\title{
High catalytic performance of palladium nanoparticles supported on multiwalled carbon nanotubes on alkene hydrogenation
}

\author{
Manuela Cano, ${ }^{a}$ Ana M. Benito, ${ }^{* a}{ }^{a}$ Wolfgang K. Maser ${ }^{a}$ and Esteban P. Urriolabeitia ${ }^{* b}$
}

The synthesis of Pd nanoparticles (Pd-NPs) supported on multiwalled carbon nanotubes (MWCNTs) and the cataytic performance of the resulting material (PdNPs/MWCNTs) on hydrogenation reactions are presented. Facile preparation approaches based on the decomposition of Pd precursors in the presence of MWCNTs lead to homogeneous dispersions of supported Pd-NPs with average size of $4 \mathrm{~nm}$ and Pd loads about $12 \%$. The catalytic performance of this material was evaluated in hydrogenation reactions of $\alpha, \beta$-unsaturated ketones, alkenes, cyclic di-, tri- and tetraenes, aromatic compounds, terpenes and terpenoids, resulting in very high activity offering short reaction times, high conversion rates, notable selectivity, and acceptable recyclabitiliy under mild conditions.

\section{Introduction}

The hydrogenation of organic molecules is probably one of the most important chemical reactions for the synthesis of new compounds. ${ }^{1}$ This process can be catalyzed homogeneously or heterogeneously, but it is well recognized that the heterogeneous version is by far more interesting from an industrial point of view, ${ }^{2}$ offering well-known benefits in terms of waste reduction, separation of the catalysts and recyclability. ${ }^{3}$ With the aim to improve efficiencies, new catalysts and supports are being developed continuously. Among the metals, palladium displays a very active catalytic behaviour. ${ }^{4}$ More specifically, palladium nanoparticles of different sizes and shapes have emerged as powerful catalytic tools. ${ }^{5,6}$ On the other hand, a large array of supports and/or stabilizers for the nanoparticles has been developed, from polymers, ${ }^{7-9}$ to ionic liquids, ${ }^{10-12}$ dendrimers, ${ }^{13}$ metallic oxides ${ }^{14,15}$ or carbonaceous materials. ${ }^{16,17}$ Among the latter, carbon nanotubes (CNTs) have received increasing attention in the last years due to their fascinating properties and excellent catalytic behavior. ${ }^{16-26}$ Their large surface/volume ratio make them as ideal supports for the incorporation of large amounts of metal using a minimum of support. However, in order to achieve a robust catalyst/support ensemble, stable under the catalytic conditions, the use of functionalized CNTs is often required. Unfortunately, the prefunctionalization of the CNTs involves numerous synthetic steps and/or tedious manipulations, which make them less attractive.

In the context of our current research about the properties of Pd-NPs deposited on MWCNTs, ${ }^{27-30}$ we have recently reported a simple and fast method to prepare the hybrid materials Pd-NPs/MWCNTs assisted by the use of microwaves, which display a high stability without prefunctionalization of the MWCNTs and an impressive catalytic activity on C-C coupling reactions (Heck, Suzuki), with full recovery of the catalyst at the end of the reaction. ${ }^{30}$ Continuing this work, in this contribution we present results on the catalytic performance and recyclability behaviour of the hybrid Pd-NPs/MWCNTs on hydrogenation reactions of a wide variety of industrially relevant substrates. The results obtained show a very high activity and notable selectivity under mild reaction conditions, avoiding the use of high pressures and/or temperatures.

\section{Results and Discussion}

Synthesis and characterization of the Pd-NP/MWCNT hybrid catalysts

In a previous communication, ${ }^{30}$ we reported that the reaction of MWCNTs with the palladium complex $\left[\mathrm{Pd}_{2}(\mathrm{dba})_{3} \mathrm{CHCl}_{3}\right](\mathrm{dba}=$ di(benzylidene)acetone) under conventional or microwave heating afforded the hybrid material Pd-NPs/MWCNTs with high loading of Pd (up to $40 \mathrm{wt} . \%$ ) in reaction times of few minutes (microwave). For this work here we reacted MWCNTs and $\left[\mathrm{Pd}_{2}(\mathrm{dba})_{3} \mathrm{CHCl}_{3}\right]$ in a 1:1 ratio for 2 min microwave heating, affording Pd-NP/MWCNT hybrid material with a Pd load of about $12.5 \mathrm{wt} . \%$. The efficiency of this reaction is about $70 \%$.

TEM images of the material (Figure 1) shows clearly that a large amount of Pd-NPs were deposited on MWCNTs. The nanoparticles are quite homogeneously distributed, and large nanoclusters were not observed. Moreover, the detailed analysis of the NPs size reveals that most of the nanoparticles are comprised in a very narrow range of 
diameters, whose average value is $4 \mathrm{~nm}$. The X-ray diffractogram (Figure 2a) is dominated by the presence of Pd (111), (200) and (220) peaks, evidencing that the Pd nanoparticles crystallized in a face-centred cubic structure. In addition the diffraction peak at $26^{\circ}$, corresponding to the graphite (002) interlayer distance, indicates the presence of MWCNTs. The Raman spectrum (Figure 2b) corroborates that the incorporation of the Pd nanoparticles to the MWCNT skeleton does not promote any important alteration of the latter, as previousy observed. ${ }^{30}$

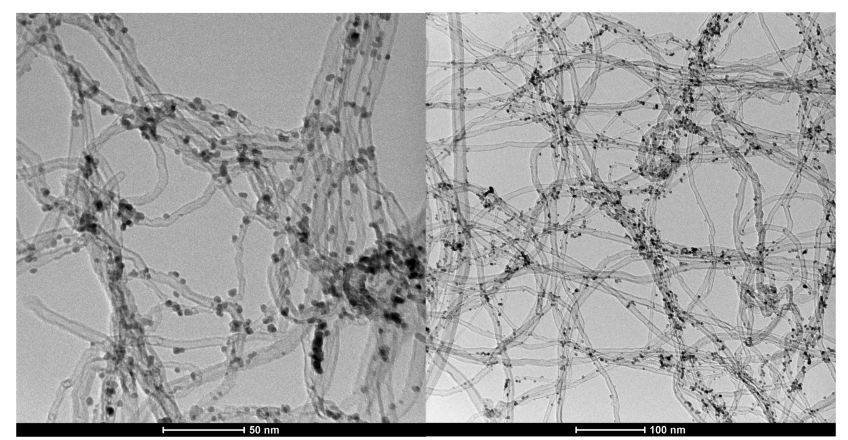

Figure 1. Two different TEM images of the Pd-NP/MWCNT material
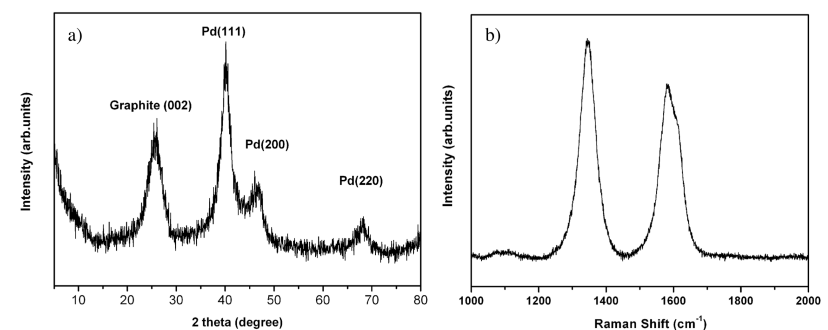

Figure 2. Characterization of the Pd-NP/MWCNT material: a) XRD spectrum; b) Raman spectrum.

We thus have achieved the synthesis of a hybrid Pd-NPs/MWCNTs material through a straightforward, facile and effective preparation route. In this material $\mathrm{Pd}$ nanoparticles with average size of $4 \mathrm{~nm}$ were homogeneously supported on the carbon nanomaterial. This Pd hybrid material was subsequently used in catalytic hydrogenation reactions as will be described in the following section.

\section{Catalytic activity of the Pd-NP/MWCNT materials for the hydrogenation of olefinic substrates.}

The behaviour of the Pd-NPs/MWCNTs materials as hydrogenation catalysts has been studied in depth. Preliminary results were promising, since a high activity was found for the hydrogenation of styrene and methylacrylate. ${ }^{30}$ Aiming to expand the scope of the reaction we have used these materials as catalysts for the hydrogenation of substrates shown in Table 1.

As a general trend, we have observed full conversions in all cases with amounts of catalyst ranging $1-5 \%$. The hydrogenation of acetophenone using $5 \%$ Pd under mild conditions $\left(\mathrm{T}=25{ }^{\circ} \mathrm{C}, \mathrm{p}_{\mathrm{H} 2}=1 \mathrm{~atm}\right)$ gives ethylbenzene (entry 1) without any detectable traces of phenylethanol. It is worth noting that these mild conditions clearly differed from the high pressures and temperatures usually needed for the hydrogenation of this type of substrates. ${ }^{31}$ When competing insaturations are present in the same sustrate the hydrogenation occurs selectively. The hydrogenation of methylvinyl ketone (entry 2) or cyclohex-2-enone (entry 3) takes place with regioselective reduction of the $\mathrm{C}=\mathrm{C}$ double bond, while the $\mathrm{C}=\mathrm{O}$ moiety remains unchanged, being this selectivity always difficult to be achieved. ${ }^{26,32,33}$ In addition to the selectivity found in these hydrogenations, we have also noticed a high activity of the PdNP/MWCNT catalysts, similar to that reported in the literature for conventional $\mathrm{Pd} / \mathrm{C}$ catalysts (same order of magnitude), and higher than that described for other systems based on SWCNTs. ${ }^{26}$ Moreover, our hydrogenation processes take place under milder reaction conditions than previously reported, which is an additional advantage. 
Table 1. Hydrogenation of relevant substrates using Pd-NP/MWCNT hybrid catalysts

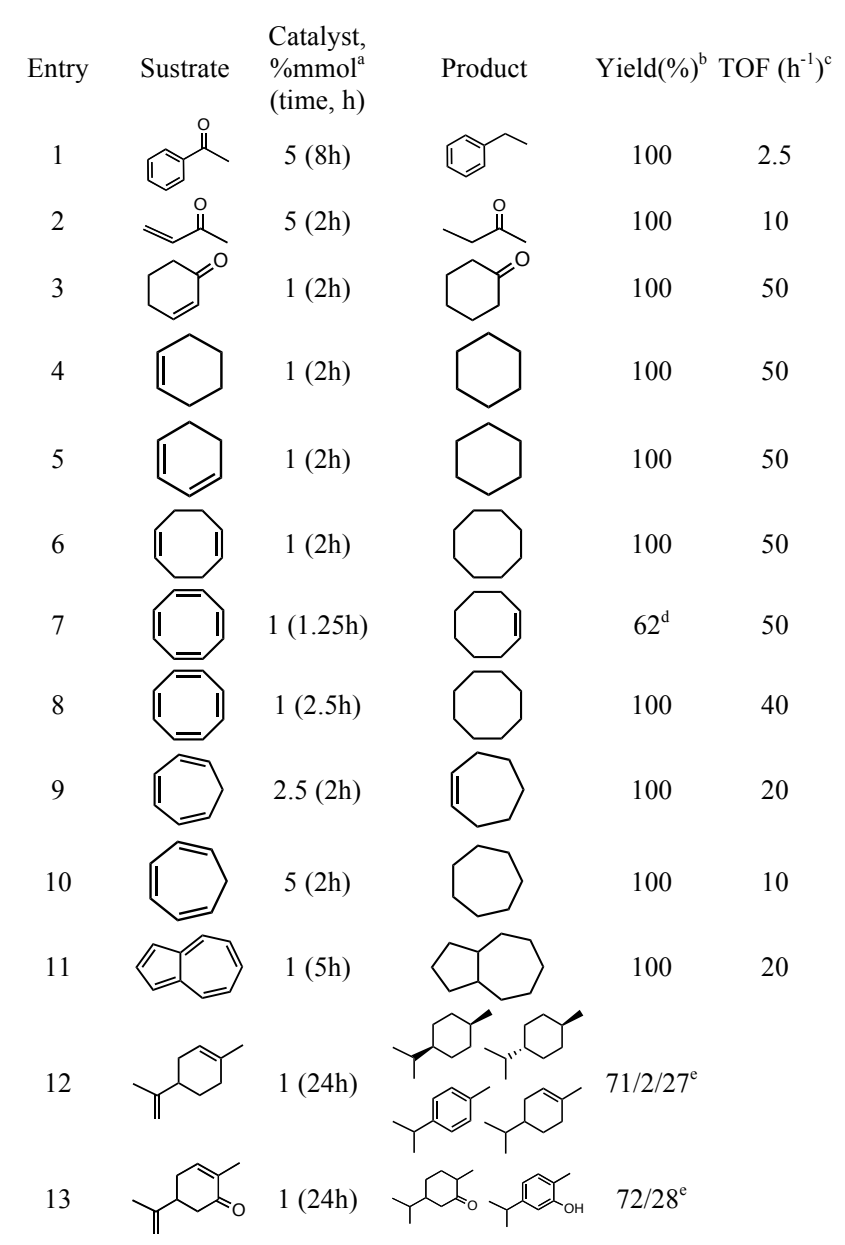

a) Catalyst $(\%)=[(\mathrm{mmol} \mathrm{Pd}) /(\mathrm{mmol}$ sustrate $)] \times 100$. The amount of the hybrid Pd-NPs/MWCNTs containing the required mmol of Pd will depend of the amount of Pd in this particular sample (tipically $12 \%$ ). b) Isolated yield. c) $\mathrm{TOF}=\mathrm{mmol}$ product $/[\mathrm{mmol}$ Pdxtime(h)]. d) the product also contains small $(<5 \%)$, but detectables, amounts of 1,5-cyclooctadiene and cyclooctane. e) molar ratios determined by integration of the corresponding peaks on the NMR spectra.

The hydrogenation of cyclohexene (entry 4) and 1,3-cyclohexadiene (entry 5) always afforded cyclohexane as the final product without detectable traces of intermediates. The hydrogenation of 1,5-cyclooctadiene (entry 6) shows again excellent conversions and selective formation of saturated cyclooctane. It is noteworthy the hydrogenation of 1,3,5,7-cyclooctatetraene (COT), which afforded almost selectively the partially hydrogenated product, cyclooctene (COE), in good yield, when short reaction times are used (entry 7). As expected, when longer reaction times are allowed the full hydrogenated derivative cyclooctane is obtained (entry 8). Although several authors report on the determination of the heat of hydrogenation of COT, ${ }^{34}$ the selective metal-catalysed hydrogenation of COT to COE has never been reported so far, to the best of our knowledge.

Even more interesting is the Pd-catalysed hydrogenation of 1,3,5-cycloheptatriene (CHT), which has been scarcely investigated. ${ }^{35}$ As can be seen, by only changing the amount of catalyst it is possible to control the targethydrogenated product: while the Pd-NP/MWCNT catalyst in a concentration of 5\% (Pd) gives the fully hydrogenated cycloheptane (entry 10), a concentration of $2.5 \%$ of Pd selectively affords cycloheptene in the same reaction times (entry 9). In both cases quantitative yields are obtained. Due to the interest of cycloheptene (raw material in organic chemistry and monomer in polymer synthesis), a further optimization was attempted. However, all changes of the reaction time and/or temperature affords higher amounts of the fully hydrogenated cycloheptane.

The hydrogenation of azulene (entry 11) affords the aliphatic decahydroazulene upon reduction of all the five $\mathrm{C}=\mathrm{C}$ bonds under mild conditions. This is noteworthy, since azulene is aromatic and, in principle, it should be more reluctant than non aromatic substrates to be hydrogenated. Moreover, in the case of acetophenone we have not 
observed hydrogenation of the arylic ring, even at the level of traces. This means that, although active enough to hydrogenate aromatic compounds, our catalysts are not able to hydrogenate the strongly aromatic benzene-like structures. Probably the different resonance energy of the azulene compared with that of benzene could account for the distinct reactivity. Although the absolute values of energy differ as a function of the bibliographic source, it seems accepted that azulene is less aromatic than benzene and that this lower aromaticity, expressed as difference in

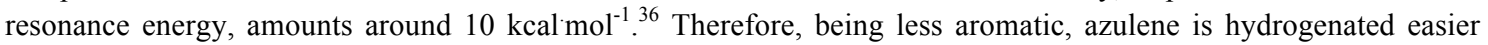
than benzene.

We have also carried out the hydrogenation of a naturally occurring product, such as $R-(+)$-limonene, a cyclic terpene. The hydrogenation of $R-(+)$-limonene (entry 12) occurs with total conversion of the starting material although, as it has been observed in preceding cases, the reaction affords mainly a mixture of three different isomers. Interestingly, the reaction occurred with some degree of selectivity, because usually up to six different products can be observed upon the hydrogenation of limonene. ${ }^{37}$ The main components of the mixture (71\%) are the two diastereoisomers of $p$-menthane (cis and trans), while the interesting $p$-menthene amounts $27 \%$, and a very small amount of $p$-cymene $(2 \%)$ is detected.

Finally, the hydrogenation of the industrially relevant $R$-carvone (entry 13) affords a mixture of cis- and transcarvomenthone and carvacrol (keto/alcohol molar ratio $=2.5 / 1)$ in $24 \mathrm{~h}$ under the usual mild conditions $\left(\mathrm{T}=25^{\circ} \mathrm{C}, \mathrm{p}_{\mathrm{H} 2}\right.$ $=1 \mathrm{~atm})$. Full conversion has been achieved, this fact being an improvement with respect to previous contributions using $\mathrm{Pd}^{7,38}$ or $\mathrm{Pt}^{38,39}$ as catalysts. Other systems have proved to be slightly more efficient, ${ }^{40,41}$ but need a somewhat more sophisticated equipment due to the use of $\mathrm{SCO}_{2}$ as solvent and high pressures. As expected, the hydrogenation of the $\mathrm{C}=\mathrm{C}$ bonds occurs preferentially, affording the cycloaliphatic ketone, while the valuable carvacrol ${ }^{41}$ results $^{2}$ from the isomerization of the starting carvone. ${ }^{38}$ Therefore, the simple catalytic procedure here described is quite active and selective, since only two of the seven possible reaction products have been obtained, with a observed molar ratio typical for this type of process. ${ }^{7,38-41}$

Another important aspect is the study of the recyclability of the catalyst. In the following we report on one representative case, namely the hydrogenation of cyclohex-2-enone (entry 3). For this purpose we have subjected the same catalyst to several consecutive batch additions of starting compound and tested the conversion for each step. After each run is completed the catalyst is isolated by filtration, washed and dried before a new batch of reactants is added. The results are collected in Table 2 . As it can be seen, the catalyst remains totally active during 4 complete runs. The activity drops during the fifth run but still gives a remarkable $60 \%$. These results are similar to those reported previously by our group for this type of Pd-NP/MWCNT catalysts, ${ }^{30}$ although referred to a Heck coupling. Therefore, the use of these materials is not limited to the first run and can be reused several times. However, further improvements in their designs are necessary in order to make them still more attractive from an applied point of view.

Table 2. Reuse of the Pd-NP/MWCNT catalyst in the hydrogenation of cyclohex-2-enone

$\begin{array}{cccccc}\text { Run } & 1 & 2 & 3 & 4 & 5 \\ \text { Conversion } & 100 & 100 & 100 & 100 & 60\end{array}$

(\%)

\section{Conclusions}

High performance catalyst based on palladium nanoparticles deposited on MWCNTs have been prepared by decomposition of $\left[\mathrm{Pd}_{2}(\mathrm{dba})_{3} \mathrm{CHCl}_{3}\right]$ in presence of MWCNTs under microwave irradiation in a simple one-pot procedure. The resulting hybrid material is characterised by $\operatorname{Pd}(0)$ nanoparticles with an average size of $4 \mathrm{~nm}$ homogeneously distributed throughout the support. Its catalytic activity has been tested in the hydrogenation of different substrates. Full conversions are achieved for a broad range of materials, such as such as $\alpha, \beta$-unsaturated ketones (acetophenone, MVK), olefins, poly-olefins (CHD, COD, CHT, COT), aromatic substrates (azulene) and natural products (limonene, carvone). Showing as well acceptable recyclability behaviour the developed catalysts may offer promise for further industrial exploitation.

\section{Experimental details}

\section{General considerations}

Solvents were dried and distilled using standard procedures before use. MWCNTs were purchased from Nanocyl Co, Belgium (Nanocyl ${ }^{\mathrm{TM}}$ NC7000, $90 \%$ carbon purity). Reagents were used as purchased from various commercial sources. The products of the catalytic reactions were characterized by NMR spectroscopy and mass spectrometry. 
The ${ }^{1} \mathrm{H}$ and ${ }^{13} \mathrm{C}\left\{{ }^{1} \mathrm{H}\right\}$ NMR spectra were recorded in $\mathrm{CDCl}_{3}$ solutions at $25^{\circ} \mathrm{C}$ on a Bruker Avance- 400 spectrometer $(\delta, \mathrm{ppm} ; \mathrm{J}, \mathrm{Hz})$, and were referenced using the solvent signal as internal standard. ESI/APCI mass spectra were recorded using an Esquire 3000 ion-trap mass spectrometer (Bruker Daltonic GmbH, Bremen, Germany) equipped with a standard ESI/APCI source. Samples were introduced by direct infusion with a syringe pump. Nitrogen served both as the nebulizer gas and the dry gas. Helium served as a cooling gas for the ion trap and collision gas for $\mathrm{MS}_{\mathrm{n}}$ experiments.

\section{Preparation of Pd-NPs/MWCNTs hybrid materials.}

Pd-NP/MWCNT catalysts were prepared using microwave assisted synthesis in a $\mathrm{CEM}^{\mathrm{TM}}$ S-class Microwave Synthesis System according to a recently published reported procedure. ${ }^{30}$ In brief, MWCNTs and the commercially available $\mathrm{Pd}$ complex $\left[\mathrm{Pd}_{2}(\mathrm{dba})_{3} \mathrm{CHCl}_{3}\right]$ were reacted in a 1:1 weight percent ratio as follows: $100 \mathrm{mg}$ of MWCNTs and $100 \mathrm{mg}$ of $\left[\mathrm{Pd}_{2}(\mathrm{dba})_{3} \mathrm{CHCl}_{3}\right]$ in $20 \mathrm{~mL}$ of freshly distilled toluene were reacted at $110{ }^{\circ} \mathrm{C}$ under Ar atmosphere applying a microwave power of 15-20 W. Reaction was completed after 2 min of microwave heating. The resulting yellow suspension was centrifuged at $4000 \mathrm{rpm}$ for $10 \mathrm{~min}$ and the precipitate was re-suspended in $20 \mathrm{~mL}$ of toluene and filtered. The black solid material was washed with $5 \mathrm{~mL}$ of $\mathrm{CH}_{2} \mathrm{Cl}_{2}$ and $15 \mathrm{~mL}$ of water, and was dried at $110{ }^{\circ} \mathrm{C}$ for one day, affording powder-like Pd-NP/MWCNT material containing about $12.5 \mathrm{wt} . \%$ of Pd.

\section{Characterization of the Pd-NPs/MWCNTs hybrid materials.}

The morphology of the prepared hybrid materials was studied by transmission electron microscopy (TEM, JEOL2000 FXII working at $200 \mathrm{kV}$ ). Samples were dispersed in ethanol in ultrasound bath for $10 \mathrm{~min}$, and a drop of the suspension was placed onto a copper grid coated with carbon film. Powder X-ray diffraction (XRD) measurements were carried out at room temperature on a Bruker D8 Advance diffractometer using a $\mathrm{Cu} \mathrm{K} \alpha \mathrm{X}$-ray radiation. The metal loading in the carbonaceous samples was determined by Inductive Coupled Plasma Spectroscopy (ICPS) using a Jobin-Ybon 2000 Ultrace Analyzer. Raman spectra were recorded with a Horiba Jobin Yvon HR800 UV spectrometer using an excitation wavelength of $532 \mathrm{~nm}$.

\section{Catalytic hydrogenation experiments.}

The Pd catalyst supported on MWCNTs, in the appropriate amount (Table 1), was dispersed in $\mathrm{CHCl}_{3}(2 \mathrm{~mL}) \mathrm{under}$ stirring in a $5 \mathrm{~mL}$ round-bottomed flask at room temperature and atmospheric pressure. To this suspension $1 \mathrm{mmol}$ of the substrate to be hydrogenated was added. The system was then evacuated and backfilled with $\mathrm{H}_{2}$ in cycles for at least five times. The pressure of $\mathrm{H}_{2}$ was kept constant $\left(\mathrm{p}_{\mathrm{H} 2}=1 \mathrm{~atm}\right)$ with a balloon system during the whole reaction time. Once the hydrogenation has been performed, the resulting suspension was filtered through a $3 \mu \mathrm{m}$ pore polycarbonate membrane filter, and the solid catalyst material was washed with $\mathrm{CH}_{2} \mathrm{Cl}_{2}$ and water, and dried at 110 ${ }^{\circ} \mathrm{C}$ overnight. The solvent was removed from the organic solution by fractional distillation, affording the hydrogenated products as oily residues, which were characterized by comparison of their ${ }^{1} \mathrm{H}$ and ${ }^{13} \mathrm{C}$ NMR spectral data with the corresponding reference samples found in the literature.

\section{Acknowledgements}

Financial support from the Spanish Ministerio de Economía y Competitividad (MINECO) and the European Regional Development Fund (ERDF) under projects CTQ2011-22589 and MAT2010-15026, CSIC under project 201080E124, and the Regional Government of Aragon and the European Social Fund (ESF) under projects (DGA-PI086-08 and E97, and DGA-ESF T66 CNN) is gratefully acknowledged. M. C. thanks MICINN and ESF for her Grant N ${ }^{\circ}$ BES2008-003503. Authors also thank Nanocyl ${ }^{\mathrm{TM}}$ (Sambreville, Belgium) for kindly providing the carbon nanotubes.

\section{Notes and references}

\footnotetext{
${ }^{a}$ Instituto de Carboquimica, ICB-CSIC, Miguel Luesma Castán 4, E-50018 Zaragoza, Spain. Fax: XX XXXX XXXX; Tel: 34976733977; E-mail: abenito@icb.csic.es

${ }^{b}$ Instituto de Síntesis Química y Catálisis Homogénea, CSIC-Universidad de Zaragoza, Pedro Cerbuna 12, E-50009 Zaragoza, Spain.Fax: 34976761187.Tel.: 34976762302; E-mail address: esteban@unizar.es.

J. F. Hartwig, Organotransition metal chemistry, from bonding to catalysis, University Science Books, 2010.

P. W. N. M. van Leeuwen, Homogeneous catalysis, understanding the art, Kluwer Academic Publishers, 2004

M. J. Climent, A. Corma and S. Iborra, Chem. Rev., 2011, 111, 1072.

J. Tsuji, Palladium reagents and catalysts, new perspectives for the 21 st century, John Wiley \& Sons, 2004.

D. Astruc, Nanoparticles and catalysis, Wiley-VCH, 2008.

I. Favier, D. Madec, E. Teuma and M. Gómez, Curr. Org. Chem., 2011, 15, 3127.

7 M. M. Dell'Ana, M. Gagliardi, P. Mastrorilli, G. P. Suranna and C. F. Nobile, J. Mol. Catal. A: Chem., 2000, $158,515$.
} 
8 T. S. Huang, Y. H. Wang, J. Y. Jiang and Z. L. Jin, Chin. Chem. Lett. 2008, 19, 102.

9 H. Hirai, H. Chawanya and N. Toshima, Bull. Chem. Soc. Jpn., 1985, 58, 682.

10 J. Dupont, G. S. Fonseca, A. P. Umpierre, P. F. P. Fichtner and S. R. Teixeira, J. Am. Chem. Soc., 2002, 124, 4228.

11 J. Durand, E. Teuma, F. Malbosc, Y. Kihn and M. Gómez, Catal. Commun., 2008, 9, 273.

12 P. Dash, S. M. Miller and R. W. J. Scott, J. Mol. Catal. A: Chem., 2010, 329, 86.

13 R. Andrés, E. de Jesús and J. C. Flores, New J. Chem., 2007, 31, 1161.

14 J. M. Nadgeri, M. M. Telkar and C. V. Rode, Catal. Commun., 2008, 9, 441.

15 A. Binder, M. Seipenbusch, M. Muhler and G. Kasper, J. Catal., 2009, 268, 150.

16 P. Serp, M. Corrias and P. Kalck, Appl. Catal. A: Gen., 2003, 253, 337.

17 R. S. Oosthuizen and V. O. Nyamori, Plat. Met. Rev., 2011, 55, 154.

18 J. K. Lee and M. J. Kim, Tetrahedron Lett., 2011, 52, 499.

19 L. Rodríguez-Pérez, C. Pradel, P. Serp, M. Gómez and E. Teuma, ChemCatChem, 2011, 3, 749.

20 G. M. Neelgund and A. Oki, Appl. Catal. A: Gen., 2011, 399, 154.

21 M. R. Nabid, Y. Bide and S. J. T. Rezaei, Appl. Catal. A: Gen., 2011, 406, 124.

22 P. Serp and E. Castillejos, Chem CatChem, 2010, 2, 41.

23 A. Jung, A. Jess, T. Schubert and W. Schütz, Appl. Catal. A: Gen., 2009, 362, 95.

24 Y. S. Chun, J. Y. Shin, C. E. Song and S. G. Lee, Chem. Commun., 2008, 942.

25 N. Karousis, G. E. Tsotsou, F. Evangelista, P. Rudolf, N. Ragoussis and N. Tagmatarchis, J. Phys. Chem. C, $2008,112,13463$.

26 A. Corma, H. Garcia and A. Leyva, J. Mol. Catal. A: Chem., 2005, 230, 97.

27 I. Sayago, E. Terrado, E. Lafuente, M. C. Horrillo, W. K. Maser, A. M. Benito, R. Navarro, E. P. Urriolabeitia, M. T. Martínez and J. Gutiérrez, Synthetic Metals, 2005, 148, 15.

28 A. Ansón, E. Lafuente, E. P. Urriolabeitia, R. Navarro, A. M. Benito, W. K. Maser and M. T. Martínez, J. Phys. Chem. B, 2006, 110, 6643.

29 A. Ansón, E. Lafuente, E. P. Urriolabeitia, R. Navarro, A. M. Benito, W. K. Maser and M. T. Martínez, J. Alloys Compd., 2007, 436, 294.

30 M. Cano, A. M. Benito, W. K. Maser and E. P. Urriolabeitia, Carbon, 2011, 49, 652.

31 J. A. Anderson, A. Athawale, F. E. Imrie, F. M. McKenna, A. McCue, D. Molyneux, K. Power, M. Shand and R. P. K. Wells, J. Catal., 2010, 270, 9

32 Y. Hu, H. Yang, Y. Zhang, Z. Hou, X. Wang, Y. Qiao, H. Li, B. Feng and Q. Huang, Catal. Commun., $2009,10,1903$.

33 S. Chandra, S. Bag, P. Das, D. Bhattacharya and P. Pramanik, Chem. Phys. Lett., 2012, 519-520, 59.

34 D. W. Roger, J. Mol. Struc., 2000, 556, 207.

35 B. D. Polkovnikov, O. M. Nefedov, E. P. Mikos and N. N. Novitskaya, Bull. Acad. Sci. USSR, Div. Chem. Sci., 1968, $17,1175$.

36 A. D. Payne and D. Wege, Org. Biomol. Chem., 2003, 1, 2383.

37 R. J. Grau, P. D. Zgolicz, C. Gutiérrez and H. A. Taher, J. Mol. Catal. A: Chem., 1999, $148,203$.

38 E. I. Klabunovskii, L. F. Godunova and L. K. Maslova, Bull. Acad. Sci. USSR, Div. Chem. Sci., 1972, $21,1020$.

39 S. R. de Miguel, M. C. Román-Martínez, D. Cazorla-Amorós, E. L. Jablonski and O. A. Scelza, Catal. Today, 2001, 66, 289.

40 C. I. Melo, R. Bogel-Lukasik, M. Gomes da Silva and E. Bogel-Lukasik, Green Chem., 2011, $13,2825$.

41 C. I. Melo, R. Bogel-Lukasik and E. Bogel-Lukasik, J. Supercrit. Fluids, 2012, 61, 191. 